\title{
Métodos para avaliação das emissões de gases do efeito estufa no sistema solo-atmosfera
}

\author{
Methods for the assessment of greenhouse gases emissions in the soil-atmosphere system
}

\author{
Falberni de Souza Costa ${ }^{1}$ Juliana Gomes ${ }^{1}$ Cimélio Bayer $^{2}$ João Mielniczuk ${ }^{2}$
}

\section{- REVISÃO BIBLIOGRÁFICA -}

RESUMO

A escolha do método para avaliar as emissões de gases do efeito estufa (GEE) é uma etapa importante para o conhecimento e/ou desenvolvimento de práticas agrícolas com potencial de mitigação do aquecimento global. A presente revisão tem por objetivo apresentar vantagens e limitações de métodos utilizados para quantificação dos fluxos de dióxido de carbono $\left(\mathrm{CO}_{2}\right)$, metano $\left(\mathrm{CH}_{4}\right)$ e óxido nitroso $\left(\mathrm{N}_{2} \mathrm{O}\right)$ no sistema solo-atmosfera. $\mathrm{O}$ balanço dos estoques de $\mathrm{C}$ orgânico no solo em sistemas conservacionistas de manejo permite avaliar o influxo líquido anual de $\mathrm{C}-\mathrm{CO}_{2}$ atmosférico no solo em comparação a sistemas de manejo convencional. Maior sensibilidade na determinação direta das emissões de $\mathrm{CO}_{2}$ in situ pode ser obtida pelo uso de câmaras sobre o solo. Nesse caso, podem ser determinadas taxas diárias com o método da captura do $\mathrm{CO}_{2}$ em solução alcalina e quantificação por titulação, e taxas horárias com o uso de analisadores automáticos de infravermelho ou cromatógrafos a gás. Pelo uso de cromatografia, é possível também a avaliação das emissões de $\mathrm{N}_{2} \mathrm{O}$ e $\mathrm{CH}_{4}$ os quais apresentam, respectivamente, potencial de aquecimento global 296 e 23 vezes superior ao do $\mathrm{CO}_{2}$. A análise dos três GEE é necessária quando se objetiva avaliar o potencial de um dado sistema de manejo na mitigação do aquecimento global, o qual pode ser expresso em $C$ equivalente.

Palavras-chave: aquecimento global, seqüestro de $C$, absorção de $\mathrm{CO}_{2}$ em soluções alcalinas, analisadores de infravermelho, cromatografia gasosa.

\section{ABSTRACT}

The sellection of the method for evaluating greenhouse gases (GHG) emissions is an important step in studies aiming at the development of agricultural practices with potential to mitigate the global warming. The objective of this review was to present advantages and disvantages of available methods to quantify fluxes of carbon dioxide $\left(\mathrm{CO}_{2}\right)$, methane $\left(\mathrm{CH}_{4}\right)$ and nitrous oxide $\left(\mathrm{N}_{2} \mathrm{O}\right)$ in the soil-atmosphere system. Carbon sequestration or annual net influx rates of $C$ $\mathrm{CO}_{2}$ in conservation tillage systems can be evaluated through the soil carbon budget. In situ evaluations can be carried out with closed chambers, where alkaline traps are used to determination of daily influxes of $\mathrm{CO}_{2}$ while gas chromatography and automatic infrared gas analyzers permit to estimate horary fluxes. In addition to $\mathrm{CO}_{2}$, gas chromatography is able to measure $\mathrm{N}_{2} \mathrm{O}$ and $\mathrm{CH}_{4}$ emissions, gases that have a global warming potential 296 and 23 times higher than $\mathrm{CO}_{2}$, respectively. Measurement of the three GHG emissions permit to estimate the net effect of soil management systems on atmosphere rodiative forcing in equivalent $C$.

Key words: global warming, $C$ sequestration, greenhouse gases, alkali $\mathrm{CO}_{2}$ absorption, infrared detectors, gas chromatography.

\section{INTRODUÇÃO}

A contribuição da agricultura para o efeito estufa antropogênico (ROBERTSON et al., 2000), pode ser reduzida, sendo o desenvolvimento de sistemas de manejo do solo com capacidade para mitigar as emissões de GEE uma importante etapa (JANZEN et al., 1998; BAYER et al., 2000). Mitigar significa intervenção antrópica que reduz as emissões ou

${ }^{1}$ Programa de Pós-graduação em Ciência do Solo, Universidade Federal do Rio Grande do Sul (UFRGS), Porto Alegre, RS, Brasil.

2Departamento de Solos, UFRGS, CP 15100, 91501-970, Porto Alegre, RS, Brasil. E-mail: cimelio.bayer@ufrgs.br. Autor para correspondência. 
aumenta os sumidouros de GEE (IPCC, 2001). O interesse nesse tema é geral e crescente na comunidade científica. Segundo previsões, em função da dependência do desenvolvimento e produtividade das culturas quanto aos fatores climáticos, a agricultura é um dos setores da atividade humana mais vulnerável aos efeitos de possíveis alterações climáticas, com repercussões negativas no que se refere à segurança alimentar em escala global (IPCC, 2001).

Das emissões totais, a agricultura contribui com aproximadamente $20 \%$ de $\mathrm{CO}_{2}$, e com $50-70 \%$ de $\mathrm{N}_{2} \mathrm{O}$ e $\mathrm{CH}_{4}$, gases cujas taxas anuais de aumento na atmosfera são de $0,5 \%, 1,0 \%$ e $0,8 \%$, respectivamente (IPCC, 2001). A previsão do impacto da elevação da temperatura em ecossistemas naturais e agroecossistemas é bastante imprecisa, mas entre algumas conseqüências, pode-se salientar a elevação em até $0,8 m$ do nível dos oceanos com desaparecimento de cidades costeiras, alternância de excessos e déficits hídricos, alteração nas áreas com aptidão agrícola de diferentes regiões do mundo, entre outros (IPCC, 2001).

O Brasil é signatário do Protocolo de Quioto, com ações já finalizadas, e outras em desenvolvimento, no cumprimento de compromissos assumidos, como é o caso, por exemplo, do inventário das emissões de GEE das atividades agropecuárias (LIMA et al., 2001), elaborado conforme recomendações do Painel Intergovernamental sobre Mudança do Clima (Intergovernmental Panel on Climate Change - IPCC). LIMA et al. (2001) destacam, entretanto, que o refinamento desse inventário dependerá de informações mais precisas fornecidas por pesquisas em âmbito nacional direcionadas à avaliação das emissões em sistemas de produção vegetal.

Em estudos desta natureza, é fundamental a definição do método a ser utilizado nas avaliações dos GEE. Nesse sentido, a presente revisão tem como principal objetivo apresentar vantagens e desvantagens de métodos disponíveis para avaliar as emissões de $\mathrm{CO}_{2}, \mathrm{~N}_{2} \mathrm{O}$ e $\mathrm{CH}_{4}$, visando fornecer orientação à pesquisadores que estão iniciando ações de pesquisa nessa importante temática.

\section{Desenvolvimento}

O desenvolvimento de estudos das emissões de GEE deve ser realizada tendo como base metodologias padronizadas de modo que os resultados gerados sejam confiáveis e permitam comparação com resultados gerados em outras regiões. A metodologia padronizada é destacadamente importante também quando do interesse de certificar atividades com potencial de redução das emissões no expressivo e emergente mercado de $\mathrm{C}$ mundial.
Na presente revisão, é apresentado inicialmente o balanço dos estoques de $\mathrm{C}$ no solo, em sistemas conservacionistas de manejo de solo como um método indireto de estimativa do influxo líquido de C atmosférico no solo, em comparação a sistemas de manejo convencional. $\mathrm{Na}$ continuidade, são apresentados métodos diretos que permitem a avaliação in situ das emissões de $\mathrm{CO}_{2}, \mathrm{CH}_{4}$ e $\mathrm{N}_{2} \mathrm{O}$ no sistema solo-atmosfera através do uso de câmaras coletoras.

Balanço dos estoques de C orgânico no solo como um método indireto para estimativa do influxo/efluxo líquido de $\mathrm{C}-\mathrm{CO}_{2}$ no sistema solo-atmosfera

O estoque de $\mathrm{C}$ orgânico do solo é determinado pelo balanço anual da adição de $\mathrm{C}$ fotossintetizado (influxo) e da perda (efluxo) de C orgânico devido a sua oxidação a $\mathrm{CO}_{2}$ pelos microrganismos heterotróficos (IPCC, 2001). Portanto, a partir da variação dos estoques de $C$ orgânico no solo, pode-se interpretar o efeito de sistemas de uso e manejo do solo quanto ao influxo ou efluxo líquido de C no sistema solo-atmosfera (JOHNSON, 1995).

Solos, sob vegetação natural, apresentam estoque de C orgânico estável, resultante da igualdade do influxo de $\mathrm{CO}_{2}$ atmosférico ao solo via plantas e do efluxo de $\mathrm{CO}_{2}$ do solo para atmosfera via decomposição microbiana. Quando o solo é cultivado, ocorre alteração na magnitude do influxo e do efluxo de $\mathrm{CO}_{2}$ no sistema solo-atmosfera, com reflexos nos estoques de $\mathrm{C}$ orgânico do solo. Normalmente, o cultivo do solo resulta na diminuição dos estoques de $\mathrm{C}$ orgânico do solo, resultante do aumento do efluxo de $\mathrm{CO}_{2}$ para atmosfera e diminuição do influxo de $\mathrm{C}$ fotossintetizado no solo. Nesse caso, a quantidade líquida de $\mathrm{CO}_{2}$ emitida do solo para atmosfera, em decorrência do uso agrícola, pode ser determinada a partir da diferença dos estoques de $\mathrm{C}$ orgânico do solo cultivado em relação ao solo sob vegetação natural. As práticas de manejo que favorecem o efluxo líquido de $\mathrm{C}$ para atmosfera são o preparo de solo intensivo com aração e gradagem e a utilização de sistemas de cultura com baixo aporte de resíduos vegetais envolvendo uso de pousio (BAYER et al., 2000; AMADO et al., 2001).

Sistemas de manejo conservacionistas determinam alterações na ciclagem de C no sistema solo-atmosfera, as quais se refletem no aumento dos estoques de C orgânico no solo (BAYER \& MIELNICZUK, 1997). O uso de sistemas de preparo sem revolvimento ou mínima mobilização do solo reduz o efluxo de $\mathrm{C}$ do solo para atmosfera devido à diminuição das taxas de decomposição da matéria orgânica (BAYER et al., 2000) e, ao se empregarem 
técnicas de rotação de culturas com alto aporte de resíduos vegetais, determina-se um aumento do influxo de C atmosférico ao solo. Neste contexto, a contribuição de sistemas de manejo conservacionistas no influxo líquido de $\mathrm{C}$ atmosférico no solo pode ser determinada a partir da diferença dos estoques de $\mathrm{C}$ orgânico no solo, em comparação ao mesmo solo submetido a um sistema convencional de manejo de solo (IPCC, 2001).

A estimativa do efluxo ou influxo líquido de C no sistema solo-atmosfera a partir dos estoques de C orgânico no solo é uma prática bastante eficiente para avaliar o efeito a longo prazo de sistemas de manejo na mitigação das alterações climáticas globais. A alteração nos estoques de $\mathrm{C}$ orgânico no solo é o procedimento indicado pelo Painel Intergovernamental sobre Mudanças Climáticas para realização de inventários das emissões de $\mathrm{CO}_{2}$ na agricultura (IPCC, 2001). Possivelmente, esse procedimento seja o mais recomendado na avaliação do seqüestro de $\mathrm{C}$ no solo sob sistemas de manejo conservacionistas, os quais têm potencial de ser uma atividade certificada de redução das emissões, de modo a participar do mercado de C estabelecido com base no Mecanismo de Desenvolvimento Limpro no âmbito do Protocolo de Quioto (IPCC, 2001).

O uso desse procedimento limita-se a experimentos de longa-duração ou áreas de lavoura com histórico conhecido, devido ao processo de acúmulo de matéria orgânica ser lento. Outro aspecto a considerar é que o uso deste método pode superestimar tanto o efluxo como o influxo de $\mathrm{C}$ quando perdas de solo por erosão ocorrem em magnitude expressiva. A superestimativa do efluxo ocorre quando um solo natural passa a ser cultivado sob manejo convencional e a diminuição dos estoques de $C$ orgânico é atribuída à sua oxidação a $\mathrm{CO}_{2}$ e liberação para atmosfera, enquanto parte deste $\mathrm{C}$ foi perdida por erosão. Por outro lado, a superestimativa do influxo pode ocorrer quando o aumento dos estoques de $\mathrm{C}$ orgânico em solo sob manejo conservacionista são calculados em comparação a um sistema de preparo convencional, que apresenta perdas expressivas de matéria orgânica por erosão. Nesses casos, devem-se utilizar áreas com perdas de solo por erosão não significativa ou descontar as perdas de $\mathrm{C}$ orgânico por esse processo.

Métodos diretos de avaliação in situ do fluxo de gases do efeito estufa

As metodologias de avaliação in situ dos fluxos de GEE no sistema solo-atmosfera demandam a utilização de câmaras, cujo o uso e implicações serão abordadas inicialmente. Posteriormente, será abordado o método da captura do $\mathrm{CO}_{2}$ em solução alcalina, bem como o uso de infravermelho e de cromatografia na quantificação das concentrações dos GEE em amostras de ar.

\section{Câmaras para amostragem de ar}

A câmara restringe a passagem de ar liberado do solo para a atmosfera, de modo que as mudanças de concentração do gás dentro da câmara possam ser medidas. Para isso, amostras de ar do interior da câmara são coletadas em intervalos de tempo previamente determinados, sendo a variação na concentração dos GEE quantificadas in situ quando do uso de sistemas automatizados, ou posteriormente, no laboratório, por infravermelho ou cromatografia gasosa. A partir da variação da concentração dos gases no tempo, estimase o efluxo ou influxo dos GEE no sistema soloatmosfera (HUTCHINSON \& LIVINGSTON, 1993).

As câmaras podem ser fechadas ou abertas. Nestas, é mantido um fluxo contínuo de ar através da câmara, com a concentração do gás atingindo uma diferença constante em relação à concentração do ar atmosférico. Nas câmaras fechadas, não há renovação do ar, sendo contínuas as mudanças de concentração do gás (MATTHIAS et al., 1980; DENMEAD \& RAUPACH, 1993).

A utilização de câmaras fechadas é mais comum. Contudo as alterações promovidas por elas no microambiente a partir de sua instalação, devem ser consideradas para a adequada interpretação dos resultados (DENMEAD \& RAUPACH, 1993; HUTCHINSON\&LIVINGSTON, 1993). HUTCHINSON \& LIVINGSTON (1993) destacam que as fontes potenciais de erro nas estimativas de fluxo com a utilização de câmaras fechadas são as perturbações físicas no ambiente, das quais se destacam as variações na temperatura, pressão e concentração dos gases dentro da câmara, e modificações na estrutura do solo. Em adição, os autores destacam o manuseio e a análise das amostras, o emprego de modelos imprecisos ou métodos inadequados para o cálculo do fluxo, além de erros com origem na amostragem por diferentes pessoas.

As dimensões e o material de construção das câmaras utilizadas para coleta das amostras de ar liberado do solo não são padronizados (FAO, 2001). Todavia, é de senso geral que elas devem ser fabricadas com materiais que não sofram degradação no campo, apresentem menor aumento da temperatura no interior da câmara, e que causem o mínimo possível de perturbações no local de instalação. Um material freqüentemente utilizado na construção de câmaras é o cloreto de polivinil (PVC), devido ao seu baixo custo 
de aquisição em relação a outros materiais, facilidade de trabalho na construção das câmaras, coloração branca (menor aquecimento), além da sua adequada durabilidade no campo.

A área de solo sob as câmaras utilizadas atualmente é geralmente inferior a $1 \mathrm{~m}^{2}$ (MATTHIAS et al., 1980; DENMEAD \& RAUPACH, 1993). Câmaras maiores tornariam mais representativas as estimativas dos fluxos, entretanto, podem ocasionar problemas de operacionalidade a campo, além de serem de custo de construção elevado. A altura da câmara, basicamente, deve ser compatível com o objetivo da pesquisa, se a avaliação é em solo desnudo ou com plantas e suas características morfológicas.

Embora as câmaras apresentem limitações relacionadas à alteração da condição real, fluxos livres entre o solo e a atmosfera, elas são ferramentas de fácil construção e podem ser utilizadas em diferentes condições de local e clima. Elas permitem ainda a detecção de fluxos de baixa magnitude e proporcionam informações sobre a variabilidade espacial das emissões. Em adição, as câmaras são particularmente úteis para estudos de processos geradores de GEE, ou efeitos nas emissões causados por modificações no ambiente solo-planta (HANSEN et al., 1993; BAGGS et al., 2002). Contudo, para avaliação precisa das emissões em escala de campo, são necessárias acima de quatro câmaras por tratamento (CLAYTON et al., 1994).

As amostras do ar incubado em câmaras fechadas podem ser coletadas manualmente, utilizandose seringa. Muitos trabalhos são realizados utilizando a mesma seringa para armazenamento da amostra até a realização da análise em laboratório. Neste caso, é importante que o material da seringa não seja permeável ao gás a ser analisado. As seringas de nylon são citadas como adequadas para coleta e armazenamento das amostras de ar, pois permitem o armazenamento da amostra, sem perdas gasosas, em até uma semana após a coleta (FERNANDES et al., 2002). As seringas de polipropileno também podem ser utilizadas para a coleta e armazenamento das amostras de ar (BALL et al., 1999; CHOUDHARY et al., 2002), sendo necessário, entretanto, que a análise do gás seja feita em 24 horas para evitar perdas dos gases a serem analisados, já que o polipropileno consiste em um material mais permeável (CHOUDHARY et al., 2002).

Alguns trabalhos de quantificação do fluxo de gases são realizados armazenando as amostras de ar em frascos de vidro previamente evacuados (KHALIL et al., 2004; SITAULA et al., 1992), os quais são mantidos fechados por uma tampa de borracha e cobertos por uma camada de silicone para evitar as perdas de amostra.
Captura de $\mathrm{CO}_{2}$ em solução alcalina

$\mathrm{O}$ monitoramento das emissões do $\mathrm{CO}_{2}$ em solos através de captura por solução alcalina é uma técnica clássica e tem sido empregada na mensuração da "respiração" do solo, basicamente de organismos (micro, meso e macro) e de raízes de plantas. No método de captura, o $\mathrm{CO}_{2}$ que difunde do solo se acumula no interior da câmara fechada, reage com a solução alcalina, contida em um recipiente dentro da câmara, formando carbonato. Portanto, denomina-se que o $\mathrm{CO}_{2}$ que reagiu e formou carbonato foi “capturado" pela solução alcalina, o qual é, posteriormente, quantificado por titulação com ácido $(\mathrm{HCl})$ da quantidade do hidróxido que não reagiu com o $\mathrm{CO}_{2}$ (ANDERSON, 1982). A solução alcalina utilizada pode ser tanto de hidróxido de sódio $(\mathrm{NaOH})$, quanto de potássio $(\mathrm{KOH})$. A utilização de um tratamento controle (sem exposição ao solo) é sugerida nesse método, cujo resultado é utilizado para correção dos resultados dos demais tratamentos avaliados. O efluxo de $\mathrm{C}-\mathrm{CO}_{2}$ é o quociente da quantidade integrada de $\mathrm{CO}_{2}$ pelo produto do tempo de exposição desse gás à solução pela área de solo sob a câmara, podendo ser expresso, por exemplo, em miligramas de $\mathrm{C}-\mathrm{CO}_{2}$ por metro quadrado por hora (ANDERSON, 1982).

A captura de $\mathrm{CO}_{2}$ em solução alcalina é o método mais antigo para estudo da emissão de $\mathrm{CO}_{2}$ de solos (LUNDERGÅRDH, 1927), e, considerando o aparato necessário, é o mais simples e barato (ANDERSON, 1982). Devido à possibilidade de sua aplicação em estudos de mudanças climáticas, o interesse neste método tem sido renovado (FREIJER \& BOUTEN, 1991; FRANZLUEBBERS et al., 1995; DUIKER \& LAL, 2000; COSTA, 2005). Esses autores utilizaram e comprovaram a aplicabilidade da captura em solução alcalina em estudos da taxa de liberação de $\mathrm{CO}_{2}$ do solo em diferentes tratamentos em condições de campo.

A precisão quantitativa do método de captura de $\mathrm{CO}_{2}$ em solução alcalina é questionada por JENSEN et al. (1996), principalmente em comparação à cromatografia e análise em infravermelho. Entretanto, em sete estudos de comparação dos métodos de captura de $\mathrm{CO}_{2}$ em solução alcalina e análise por infravermelho, em um total de nove, ROCHETTE et al. (1997) obtiveram resultados comparáveis de efluxo de $\mathrm{CO}_{2}$ entre esses métodos. Nos outros dois estudos, as medidas com o método da captura foram cerca de $22 \%$ menores do que com o analisador de infravermelho. Estes autores mencionam que estudos para entender as causas do efluxo menor pelo método da captura podem ajudar a melhorar o método, e que o método é uma alternativa boa quando uma estimativa integrada do efluxo médio durante um período de tempo atende os objetivos da pesquisa. 
A utilização do método de captura permite resultados precisos em avaliações com tempo de exposição superiores a 4 horas. Salienta-se que isso é possível no sistema de câmara fechada porque não ocorre aumento da concentração de $\mathrm{CO}_{2}$ dentro da câmara com o tempo, devido à sua reação com a solução alcalina, observado o aspecto de eficiência de reação da solução alcalina, comentado a seguir. $\mathrm{O}$ aumento da concentração do gás na câmara diminuiria a difusão do gás do interior do solo, sendo esse um dos principais fatores que limita o tempo em que a câmara deve permanecer fechada sobre o solo, sobretudo em estudos em que as amostras de ar são coletadas para análise por cromatografia gasosa.

Um aspecto a ser considerado nesse método é a eficiência da solução alcalina em reagir com o $\mathrm{CO}_{2}$ na mesma velocidade com que ele está sendo liberado do solo, o que está, por sua vez, relacionado à concentração da solução alcalina utilizada, ao tempo de exposição à solução alcalina, à temperatura no interior da câmara, e à relação entre as áreas de solo sob a câmara e do recipiente que contém a solução alcalina (ANDERSON, 1982). Outra limitação do método é quando se deseja estimar fluxos em período de tempo inferior a 4 horas (ROCHETTE et al., 1997).

\section{Analisadores de infravermelho}

O princípio da quantificação utilizando detectores de infravermelho é baseada na capacidade que os gases de $\mathrm{CO}_{2}, \mathrm{CH}_{4}$ e $\mathrm{N}_{2} \mathrm{O}$ possuem de absorver a radiação eletromagnética na faixa do infravermelho. Trabalhos recentes mostram a possibilidade da utilização de espectrômetros de Infravermelho por Transformada de Fourier (Fourier Transform Infrared Spectroscopy -FTIR) para quantificação dos GEE (GRIFFITH \& GALLE, 2000; GRIFFITH et al., 2002; HEDGE et al., 2003). A quantificação dos gases por FTIR é obtida relacionando a concentração com os valores da absorbância nos seguintes comprimentos de onda: 2239 e $2393 \mathrm{~cm}^{-1}$ para o $\mathrm{CO}_{2}, 2999 \mathrm{~cm}^{-1}$ para o $\mathrm{CH}_{4}$ e entre 2214 a $2236 \mathrm{~cm}^{-1}$ para o N $\mathrm{N}_{2} \mathrm{O}$ (HEGDE et al., 2003).

A quantificação dos GEE em amostras de ar por FTIR pode ser realizada em laboratório, utilizando aparelho de bancada, ou ainda in situ (HEDGE et al., 2003). Para o caso de ser realizada a quantificação em laboratório, a amostragem é realizada empregando o método da câmara fechada, conforme descrito anteriormente. Segundo GRIFFITH \& GALLE (2000), a utilização do FTIR para quantificação é uma técnica muito prática para as estimativas de fluxos dos GEE, pois é possível quantificar os três gases em uma única amostragem. Devido à alta precisão analítica e sensibilidade exigidas para os gases $\mathrm{N}_{2} \mathrm{O}$ e $\mathrm{CH}_{4}$, o que requer o desenvolvimento de aparelhos mais estáveis e com melhor controle de pressão e temperatura, o emprego mais comum de detectores de infravermelho é para quantificação do fluxo de $\mathrm{CO}_{2}$. Os fluxos de $\mathrm{CO}_{2}$ estão na ordem de ppm e, portanto estão de acordo com o limite de detecção do FTIR.

Em vários trabalhos, a quantificação do fluxo de $\mathrm{CO}_{2}$ é realizada com analisadores automáticos com detector de infravermelho (LA SCALLA et al., 2000), disponíveis no mercado. O analisador automático de infravermelho comercial apresenta faixa de detecção entre 0 a 3000ppm. A quantificação do fluxo é realizada in situ. Uma das principais características e vantagens quando do analisador automático é quanto a sua aquisição de dados. As medidas de concentração de $\mathrm{CO}_{2}$ emitido pelo solo são obtidas em frações de minuto, originando um contínuo monitoramento do aumento da concentração deste gás. Desta maneira, é possível obter um bom ajuste de regressão entre as variáveis concentração e tempo, resultando em uma avaliação de fluxo bastante precisa e adequada para avaliações em curtos períodos de tempo (DAVIDSON et al., 2002). Em analisadores automáticos de infravermelho a câmara de incubação do ar emitido pelo solo funciona como uma câmara aberta. Neste sistema, o equipamento realiza o ajuste da pressão dentro da câmara , evitando a influência da pressão no aumento da concentração de $\mathrm{CO}_{2}$ no interior desta, e conseqüentemente, evitando valores superestimados de influxo deste gás. Outras interferências como a presença do vapor de água dentro da câmara, também são contabilizados para medida de fluxo do $\mathrm{CO}_{2}$, o que aumenta ainda mais a sensibilidade e precisão do aparelho. A preocupação com o aumento da concentração dos GEE na atmosfera, em especial tendo atividades agrícolas como fontes, tem exigido estudos com mais precisão nas estimativas realizadas a campo, a qual é obtida quando do uso deste equipamento devido a sua adequada sensibilidade de detecção (REICOSKY \& LINDSTROM, 1993; JENSEN et al., 1996) e, também adequada precisão, em função da correção da influência da câmara sobre fatores como temperatura do ar e do solo, umidade do solo, insolação, entre outros (LA SCALLA, et al., 2000; LA SCALLA et al., 2001).

As principais vantagens do uso do analisador automático são a sua precisão, sensibilidade e versatilidade. A metodologia permite a aquisição de dados in situ, com leituras rápidas e precisas, aspectos importantes para estudos a campo. A limitação do método é a exclusiva leitura de $\mathrm{CO}_{2}$, além de seu custo elevado. Pode-se avaliar os demais GEE $\left(\mathrm{N}_{2} \mathrm{O}\right.$ e $\left.\mathrm{CO}_{2}\right)$ por detectores de infravermelho utilizados em análises 
de laboratório. Tendo em vista esta prática e precisa avaliação dos fluxos in situ, seria relevante o desenvolvimento de analisadores automáticos que permitissem as medidas dos fluxos dos três GEE.

\section{Cromatografia gasosa}

Os componentes principais de um sistema cromatográfico são o gás carregador, a coluna cromatográfica, o detector e o sistema computacional para processamento dos dados. O planejamento de um sistema cromatográfico deve ser orientado em função do gás que se deseja quantificar, a faixa de concentração que este se apresenta na amostra e quais outros componentes presentes dos quais se precisa separálo. A versatilidade de um sistema cromatográfico permite escolher adequadamente os componentes e montá-los em um só aparelho para quantificar os três GEE. Vale salientar que isto é uma opção para quantificação dos gases. A quantificação por cromatografia gasosa pode ser realizada em equipamentos diferentes, o que é mais comumente encontrado em laboratório.

A quantificação por cromatografia exige a presença de detector adequado e sensível o suficiente para o gás que se deseja analisar. $\mathrm{O}$ gás $\mathrm{CO}_{2}$ pode ser quantificado em cromatógrafos equipados com detector de condutividade térmica (Thermal Conductivity Detector - TCD) (BRAITHAWAITE \& SMITH, 1999; BALL et al., 1999; SITAULA et al., 1992) e de captura de elétrons (ECD - Electron Capture Detector) (RUSER et al., 1998; FERNANDES et al., 2002; FLESSA et al., 2002). O detector de TCD é considerado detector universal, mas sua sensibilidade é baixa (ppm), e, portanto não é adequado para a quantificação de $\mathrm{N}_{2} \mathrm{O}$ e $\mathrm{CH}_{4}$ que estão presentes na atmosfera na ordem de ppb. O óxido nitroso é usualmente quantificado por detector ECD (CHENG et al., 2004; FERNANDES et al., 2002; BAGGS et al., 2002) Logo, quando se utiliza detector de ECD tem-se a possibilidade de quantificar tanto o gás $\mathrm{N}_{2} \mathrm{O}$ quanto o $\mathrm{CO}_{2}$. Já para o $\mathrm{CH}$ é utilizado o detector de ionização de chama (FID - Flame Induced Detector) quando se utiliza amostras de ar atmosférico (SITAULA et al., 1992; FERNANDES et al., 2002; FLESSA et al., 2002; RUSER et al., 1998).

A coluna e o gás de arraste de um sistema cromatográfico devem ser eficientes para a separação dos compostos a serem quantificados. A coluna em um sistema cromatográfico tem a função de determinar retenção dos compostos na coluna de modo que cada composto alcance o detector em diferentes tempos (tempo de retenção). O gás de arraste deve ser quimicamente inerte e com baixo teor de contaminante, podendo ser utilizado hélio, nitrogênio ou hidrogênio.
Em cromatografia, existe a possibilidade de montar sistemas com colunas capilares "megabore”, ou colunas empacotadas. Na literatura, observa-se maior utilização de colunas empacotadas (BALL et al., 1999; CHENG et al., 2004; FERNANDES et al., 2002) e megabore (BAGGS et al. 2002; SITAULA et al., 1992) na quantificação dos GEE. A coluna empacotada apresenta menor custo em relação a "megabore”. Entre as colunas empacotadas a mais utilizada é a PORAPAKQ (CHENG et al., 2004), que apresenta eficiência para a separação dos três gases.

Um cromatógrafo pode ser equipado com três detectores (TCD, ECD e FID) ou, alternativamente, com dois detectores (ECD e FID), sendo que as duas situações possibilitam a quantificação do $\mathrm{CO}_{2}, \mathrm{CH}_{4} \mathrm{e}$ $\mathrm{N}_{2} \mathrm{O}$ em amostras de ar (SITAULA et al., 1992; LOFTFIELD et al., 1997). Porém, em muitos trabalhos, a quantificação dos gases é realizada em cromatógrafos separados. Para os dois casos, têm-se qualidade adequada de análise utilizando as condições cromatográficas adequadas. A utilização de colunas megabore, na maioria das vezes, requer sistemas cromatográficos mais sofisticados, apresentando précolunas e "back-flush” (SITAULA et al., 1992).

O procedimento de coleta de gás para análise por cromatografia gasosa (CG), geralmente é realizado com a utilização da câmara fechada. São coletadas várias amostras do ar incubado em um determinado intervalo de tempo. A coleta e o armazenamento das amostras são realizados com seringas ou frascos de vidro.

A CG apresenta-se como técnica já consolidada para quantificação das emissões do três GEE, (LINN \& DORAN, 1984; LOFTIELD et al., 1997; KESSAVALOU et al., 1998; MOSIER, et al., 1998; BALL et al., 1999). Já que a avaliação dos três gases é importante, a técnica de CG, por ser já consolidada, oferece maior praticidade para seus usuários, tendo em vista a maior disponibilidade do apoio técnico na manutenção de cromatógrafos.

\section{CONCLUSÕES}

Os métodos do balanço dos estoques de $\mathrm{C}$ orgânico no solo e a captura do $\mathrm{CO}_{2}$ em soluções alcalinas são exclusivos para o estudo dos fluxos anuais e diários de $\mathrm{CO}_{2}$, respectivamente. Fluxos de $\mathrm{CO}_{2}$ também podem ser obtidos com analisadores automáticos de infravermelho e por cromatografia gasosa, métodos indicados para estudos com exigência de maior sensibilidade e com objetivo de avaliar taxas de emissões em curtos espaços de tempo. Além do $\mathrm{CO}_{2}$, a cromatografia gasosa permite a análise de $\mathrm{N}_{2} \mathrm{O}$ e 
$\mathrm{CH}_{4}$, o que permite calcular o impacto efetivo de práticas de manejo de solo no forçamento radiativo da atmosfera. Os resultados podem ser expressos em $\mathrm{C}$ equivalente $\mathrm{e}$ podem ser calculados a partir das emissões e respectivos potenciais de aquecimento global $\left(\mathrm{CO}_{2}=1\right.$, $\mathrm{CH}_{4}=23$, e $\mathrm{N}_{2} \mathrm{O}=296$ ) dos três principais gases do efeito estufa com origem na agricultura.

\section{REFERÊNCIAS}

AMADO, T.J.C et al. Potencial de culturas de cobertura em acumular carbono e nitrogênio no solo no plantio direto e a melhoria da qualidade ambiental. Revista Brasileira de Ciência do Solo, v.25, p.189-197, 2001.

ANDERSON, J.P.E. Soil respiration. In: PAGE, A.L. et al. (ed). Methods of soil analysis. Part 2. 2.ed. Madison, WI ASA, 1982. p.837-871. (Agron Monogr 9).

BAGGS, E.M. et al. Nitrous oxide release from soils receiving $\mathrm{N}$-rich crop residues and paper mill sludge in eastern Scotland. Agriculture, Ecosystems and Environment, v.90, p.109123, 2002.

BALL, B.C. et al. Field $\mathrm{N}_{2} \mathrm{O}, \mathrm{CO}_{2}$ and $\mathrm{CH}_{4}$ fluxes in relation to tillage, compaction and soil quality in Scotland. Soil \& Tillage Research, v.53, p.29-39, 1999.

BAYER, C.; MIELNICZUK, J. Características químicas do solo afetadas por métodos de preparo e sistemas de culturas. Revista Brasileira de Ciência do Solo, v.21, p.105-112, 1997.

BAYER, C. et al. Efeito de sistemas de preparo e de cultura na dinâmica da matéria orgânica e na mitigação das emissões de $\mathrm{CO}_{2}$. Revista Brasileira de Ciência do Solo, v.24, p.599$607,2000$.

BRAITHWAITE, A.; SMITH, F.J. Chromatographic methods. 5.ed. Dordrecht: Kluwer, 1999. p.165-257.

BURFORD, J.R.; BREMNER, J.M. Gas chromatographic determination of carbon dioxide evolved from soils in closed systems. Soil Biology and Biochemistry, v.4, p.191-197, 1972.

CHENG, W. et al. $\mathrm{N}_{2} \mathrm{O}$ and $\mathrm{NO}$ production in various chinese agricultural soils by nitrification. Soil Biology Biochemistry, v.36, p.953-963, 2004.

CLAYTON, H. et al. Measurement of nitrous oxide emissions from fertilized grassland using closed chambers. Journal of Geophysical Research, v.99, p.16599-16607, 1994.

COSTA, F. Estoques de carbono orgânico e efluxos de dióxido de carbono e metano de solos em preparo convencional e plantio direto no subtrópico brasileira. 2005. 145f. Tese (Doutorado em Ciência do Solo) - Curso de Pós-graduação em Solos, Universidade do Rio Grande do Sul.

CHOUDHARY, H.A. et al. Nitrous oxide emissions from New Zealand cropped soil; tillage effects, spatial and seasonal variability. Agriculture, Ecosystems and Environment, v.93, p.33-43, 2002.
DAVIDSON, E.A. et al. Minimizing artifacts and bases in chamber-based measurement of soil respiration. Agricultural and Forest Meterology, v.113, p.21-37, 2002.

DENMEAD, O.T.; RAUPACH, M.R. Methods for measuring atmospheric gas transport in agricultural and forest systems. In: HARPER, L.A. et al. (ed). Agricultural ecosystem effects on trace gases and global climate change. Madison: ASA, CSSA e SSSA, 1993. p.19-43. (ASA Spec. Publ. 55).

DUIKER, S.W; LAL, R. Carbon budget study using $\mathrm{CO}_{2}$ flux measurements from a no till system in central Ohio. Soil \& Tillage Research, v.54, p.21-30, 2000.

FAO. Global estimates of gaseous emissions of $\mathrm{NH}_{3}$, $\mathrm{NO}$ and $\mathrm{N}_{2} \mathrm{O}$ from agricultural land. Rome, 2001. 106p.

FERNANDES, S.A.P. et al. Seasonal variation of soil chemical properties and $\mathrm{CO}_{2}$ and $\mathrm{CH}_{4}$ fluxes in unfertilized an P-fertilizes pastures in an Ultisol of the Brazilian Amazon. Geoderma, v.107, p. 227-241, 2002

FLESSA, $\mathrm{H}$. et al. $\mathrm{N}_{2} \mathrm{O}$ and $\mathrm{CH}_{4}$ fluxes in potato fields: automated measurement, management effects and temporal variation Geoderma, v.105, p.307-325, 2002

FRANZLUEBBERS, A.J. et al. Tillage-induced seasonal changes in soil physical properties affecting soil $\mathrm{CO}_{2}$ evolution under intensive cropping. Soil \& Tillage Research, v.34, p.4160, 1995.

FREIJER, J.I.; BOUTEN, W. A comparison of field methods for measuring soil carbon dioxide evolution: experiments and simulation. Plant Soil, v.135, p.133-142, 1991.

GRIFFITH, D.W. et al. Air-land use exchanges of $\mathrm{CO}_{2}, \mathrm{CH}_{4} \mathrm{e}$ $\mathrm{N}_{2} \mathrm{O}$ measured by FTIR spectrometry and micrometeorological techniques. Atmosphere \& Environment, v.36, p.1833-1842, 2002.

GRIFFITH, D.W.T.; GALLE, B. Flux measurements of $\mathrm{NH}_{3}$, $\mathrm{N}_{2} \mathrm{O}$ and $\mathrm{CO}_{2}$ by dual-beam FTIR spectroscopy and flux-gradient technique. Atmospheric Environment, v.34, p.1087-1098, 2000 .

HANSEN, S. et al. $\mathrm{N}_{2} \mathrm{O}$ and $\mathrm{CH}_{4}$ fluxes in soil influenced by fertilization and tractor traffic. Soil Biology and Biochemistry, v.25, p.621-630, 1993.

HEGDE, U. et al. Methane and carbon dioxide emissions from Shan-Chu-Ku landfill site in northern Taiwan. Chemosphere, v.52, p.1275-1285, 2003

HUTCHINSON, G.L.; LIVINGSTON, G.P. Use of chamber systems to measure trace gas fluxes. In: HARPER, L.A. et al. (ed). Agricultural ecosystem effects on trace gases and global climate change. Madison: ASA, CSSA e SSSA, 1993. p.63-78. (ASA Spec. Publ. 55).

IPCC - Intergovernmental Panel on Climate Change. Climate change 2001: the scientific basis. United Kingdom: Cambridge University, 2001. 881p.

JANZEN, H.H. et al. Management effects on soil C storage on the Canadian prairies. Soil \& Tillage Research, v.47, p.181195, 1998 
JENSEN, I.S. et al. Soil surface $\mathrm{CO}_{2}$ flux as na index of soil respiration in situ: a comparison of two chamber methods. Soil Biology and Biochemistry, v.28, p.1297-1306, 1996.

JOHNSON, M.G. The role of soil management in sequestering soil carbon. In: LAL, R. et al. (eds). Soil management and greenhouse effect. Advances in soil science. Boca Raton: CRC, 1995. p.351-362.

KHALIL, M. et al. Nitrous oxide production by nitrification and denitrification in soil aggregates as affected by $\mathrm{O}_{2}$ concentration. Soil Biology and Biochemistry, v.36, p.687699, 2004.

KESSAVALOU, A. et al. Greenhouse gas fluxes following tillage and wetting in a wheat-fallow cropping system. Journal of Environmental Quality, v.27, p.1105-1116, 1998.

LA SCALA Jr. et al. Carbon dioxide emission related to chemical properties of a tropical bare soil. Soil Biology and Biochemistry, v.32, p.1469-1473, 2000.

LA SCALA Jr. et al. Carbon dioxide emissions after application of tillage systems for a dark red latosol in southern Brazil. Soil \& Tillage Research, v.62, p.163-166, 2001.

LIMA, M.A. et al. Estimativa das emissões de gases do efeito estufa provenientes de atividades agrícolas no Brasil. In: LIMA et al. (org). Mudanças climáticas globais e a agropecuária brasileira. Jaguariúna, SP: EMBRAPA Meio Ambiente, 2001. p.169-189.

LINN, D.M.; DORAN, J.W. Effect of water-filled pore space on carbon dioxide and nitrous oxide production in tilled and notilled soils. Soil Science Society of American Journal, v.48, p.1267-1272, 1984
LOFTFIELD, N. et al. Automated gas chromatographic system for rapid analysis of the atmospheric trace gases methane, carbon dioxide, and nitrous oxide. Journal of Environmental Quality, v.26, p.560-564, 1997.

LUNDEGÅRDH, H. Carbon dioxide evolution of soil and crop growth. Soil Science, v.23, p.417-453, 1927.

MATTHIAS, A.D. et al. A simple chamber technique for field measurement of emissions of nitrous oxide from soils. Journal of Environmental Quality, v.9, p.251-256, 1980.

MOSIER, A.R. et al. Methane and nitrous oxide fluxes in an acid oxisol in western Puerto Rico: effect of tillage, liming and fertilization. Soil Biology and Biochemistry, v.30, p.2087-2098, 1998.

REICOSKY, D.C.; LINDSTROM, M.J. The effect of fall tillage method on short-term carbon dioxide flux from soil. Agronomy Journal, v.85, p.1237-1243, 1993.

ROBERTSON, G.P. et al. Greenhouse gases in intensive agriculture: contributions of individual gases to the radiative forcing of the atmosphere. Science, v.289, p.1922-1925, 2000.

ROCHETTE et al. Description of a dynamic closed chamber for measuring soil respiration and its comparison with others techniques. Canadian Journal of Soil Science, v.77, p.195203, 1997.

RUSER, R. et al. Compaction and fertilization effects on nitrous oxide and methane fluxes in potato fields. Soil Science Society American Journal, v.62, p.1587-1595, 1998.

SITAULA, B.K. et al. Rapid analysis of climate gases by wide bore capillary gas chromatography. Journal of Environmental Quality, v.21, p.493-496, 1992. 